\title{
The NTI-tss device may be used successfully in the management of bruxism and TMD
}

\author{
Abstracted from \\ Stapelmann H, Türp JC. \\ The NTI-tss device for the therapy of bruxism, temporomandibular disorders, and headache - \\ where do we stand? A qualitative systematic review of the literature. BMC Oral Health 2008 29; 8: 22. \\ Address for correspondence: Jens C. Türp, Clinic for Reconstructive Dentistry and Temporomandibular Disorders, \\ Dental School, Hebelstrasse 3, 4056 Basel, Switzerland. E-mail: jens.tuerp@unibas.ch
}

\section{Question: Is the NTI-tss device effective in the treatment of temporomandibular disorders and bruxism?}

Data Sources The Cochrane Library, Medline, TRIP database, MEDPILOT. DE, BIREME, several German-language journals, Google Scholar, the Web of Science, NTI-tss-associated websites, references of relevant articles and the MAUDE database of adverse events.

Study selection Randomised controlled trials (RCT) were included and their quality was assessed using the Jadad score.

Data extraction and synthesis A qualitative synthesis was carried out. Results Nine out of 68 relevant publications reported the results of five different RCT. Two RCT concentrated on electromyographic (EMG) investigations in patients with temporomandibular disorders (TMD) and concomitant bruxism or with bruxism alone. In both studies, the NTI-tss device showed significant reduction of EMG activity compared with use of an occlusal stabilisation splint. Two RCT focused exclusively on TMD patients: in one, a stabilisation appliance led to greater improvement than use of an NTI-tss device, whereas in the other no difference was found. In a further RCT, participants who suffered from tension-type headaches or migraines responded more favourably to the NTI-tss splint than to a bleaching tray. NTI-tss-induced complications related predominantly to single teeth or to the occlusion.

Conclusions Evidence from RCT suggests that the NTI-tss device may be successfully used for the management of bruxism and TMD. To avoid potential unwanted effects, it should be chosen only if the practitioner is certain a patient will be compliant with follow-up appointments. The NTI-tss bite splint may be justified when a reduction of jaw closer muscle activity (eg, jaw clenching or tooth grinding) is desired, or as an emergency device when people have acute temporomandibular pain and, possibly, restricted jaw opening.

\section{Commentary}

The NTI-tss splint has been marketed for about 10 years. The manufacturer and their distributors claim that the splint can be used in the prophylactic treatment of migraine and migraine-associated tension-type headache as well as to prevent bruxism and "temporomandibular joint syndrome". The biological effects are claimed to be associated with a reduction

Stapelmann and Türp set out to document the effectiveness and efficacy of the NTI-tss splint. They used an impressive and exhaustive search in multiple and multilingual bibliographic databases, so there is little reason to assume that any trials have escaped their scru- tiny. The yield of only five RCT from 68 eligible publications is therefore astonishingly small. Their search strategies (Tables 1 to 9 of the review) indicate that the authors aimed to document the intervention principally in people who have TMD. However, they also attempted to identify other studies, without success, that addressed alleged effectiveness against migraine and migraine-associated, tension-type headache as well as against bruxism (as independent from TMD).

The five RCT that were included ranged from poor to high methodological quality, but they all had rather small sample sizes. According to the review authors, the one study that had a slightly larger study sample, of 94 migraine patients, had multiple methodological flaws. In retrospect, it is perhaps unfortunate that this particular paper, according to this author's (www.drshankland.com) and manufacturer's websites, formed the basis for the US Federal Drug Administration's decision to approve the marketing of this device for use by people with migraine and migraine-associated headaches, bruxism, and TMD.

No meta-analyses were carried out because of the heterogeneity of participant samples, indications for splint therapy, and differences in reported surrogate and clinical outcomes.

A secondary aim was to identify whether adverse events following the use of this particular splint had been reported. They concluded that possible side effects, particularly those related to the teeth and the occlusion, remain the greatest concern when wearing NTI-tss splints, even though according to published reports the prevalence and severity of adverse events following the use of the NTI-tss splint seem to be minimal and the concern about risk of aspiration has not been corroborated by any published reports. It should be taken into consideration, however, that reports of side effects of intra-oral devices seldom appear as case reports in the literature and, thus, the reported incidence of aspiration or swallowing of foreign bodies of dental origin varies considerably in the literature. ${ }^{1}$

In conclusion, the authors suggest that use of the NTI-tss splint may be justified in certain circumstances. It is this commentator's opinion that the scarcity of clinical data demonstrated here requires a cautious approach to use of this device. With this, and other devices continuously arriving on the market, we should wait for evidence of efficacy and effectiveness from the manufacturer rather than conducting field experiments with our patients.

Asbjørn Jokstad

Faculty of Dentistry, University of Toronto, Toronto, Canada

1. Dibiase AT, Samuels RH, Ozdiler E, Akcam MO, Turkkahraman H. Hazards of orthodontics appliances and the oropharynx. J Orthodont 2000; 27: 295-302.

Evidence-Based Dentistry (2009) 10, 23. doi:10.1038/sj.ebd.6400635 